\title{
The efficacy of single posterior debridement, bone grafting, and instrumentation for the treatment of thoracic spinal tuberculosis
}

Jiao Zhou

Shaanxi Provincial People's Hospital

Quanyi Li

Shaanxi Provincial People's Hospital

Yongchun Zhou (D Yong_chunzhou@163.com )

Shaanxi Provincial People's Hospital

Qichun Song

Shaanxi Provincial People's Hospital

Research article

Keywords: thoracic, spinal tuberculosis, single anterior, single posterior, debridement

Posted Date: March 26th, 2020

DOI: https://doi.org/10.21203/rs.3.rs-19393/v1

License: (c) (i) This work is licensed under a Creative Commons Attribution 4.0 International License.

Read Full License 


\section{Abstract}

Background: The aim of this study was to investigate the clinical efficacy of single posterior debridement, bone grafting, and instrumentation for the treatment of thoracic spinal tuberculosis of adult patients.

Methods: A retrospective analysis was conducted on 88 adult patients with thoracic spinal tuberculosis between June 2013 and September 2017. All patients were treated with single posterior debridement, bone grafting and instrumentation. The clinical manifestations and laboratory and imaging results of the approach were subsequently analysed.

Results: All patients were followed up for $40.6 \pm 4.1 \mathrm{~m}$ (range, 36-48 m). Bony fusion was achieved in all bone grafts. The VAS scores, ESR, and CRP levels 6 weeks after operation and at the final follow-up were significantly lower than the preoperative levels $(P<0.05)$. The postoperative and final-follow-up kyphosis angles were both significantly smaller than the pre-operative kyphosis angles $(P<0.05)$. The postoperative angle correction rate reached $81.5 \%$, and the postoperative angle loss only reached $4.1 \%$. At the last follow-up, ASIA improvement was significant compared with the preoperative levels $(P<0.05)$.

Conclusion: The single posterior approach can achieve satisfactory clinical outcomes in the treatment of thoracic spinal tuberculosis.

\section{Introduction}

The incidence of spinal tuberculosis (TB) is increasing in developing countries, and every year two to three million deaths worldwide are related to spinal TB [1-3]. Spinal TB is the most frequent skeletal tuberculosis, accounting for almost $50 \%$ of all skeletal tuberculosis cases [4]. Spinal infections can cause destruction and collapse of the vertebral body and further lead to kyphosis and neurological impairment [5]. When the blood supply to the thoracic spinal cord is inadequate because of thoracic spinal canal stenosis, patients suffering from severe destruction of bone or spinal instability are more prone to neurological impairment $[6,7]$.

Although chemotherapy plays a crucial role in spinal TB treatment, it is often necessary to correct kyphosis and improve neurological function through surgical intervention. Debridement, kyphosis correction, and reconstruction of spinal stability are widely used as a method of surgical treatment for spinal tuberculosis [8]. The optimal surgical treatment for spinal TB remains controversial. The single anterior approach allows for direct debridement, bone grafting, and instrumentation, yet the outcomes of this approach regarding kyphosis correction and maintenance are far from satisfactory $[8,9]$. The single posterior approach is a proven spinal TB treatment with favourable outcomes [10,11]; however, it still fails to achieve thorough debridement of lesions in the anterior spine.

In this study, an analysis was performed of the clinical outcomes of single posterior debridement, bone grafting, and instrumentation in treating adult patients with thoracic spinal TB. 


\section{Patients And Methods \\ Basic information}

This retrospective study included 88 active pulmonary tuberculosis-free patients who were diagnosed with active spinal TB between June 2013 and September 2017. Clinical diagnosis of active spinal TB was based on clinical features, investigations [high erythrocyte sedimentation rate (ESR), and C-reactive protein (CRP)], radiographic examination [x-ray, computed tomography (CT), and magnetic resonance imaging (MRI)], and pathological examination. Other inclusion criteria were as follows: (1) monosegmental thoracic spinal TB, with the destruction limited to a single vertebral segment and affecting no more than one vertebral motor unit; (2) progressive neurological impairment; (3) poor outcomes of conservative treatment; (4) serious kyphosis or progression of kyphosis. The exclusion criteria consisted of (1) postoperative recurrence of thoracic spinal TB; (2) thoracic spinal TB combined with serious osteoporosis; (3) thoracic spinal TB combined with cancer or degeneration of intervertebral disc that might affect the evaluation of clinical outcomes; and (4) thoracic spinal TB combined with severe tuberculosis in other organs (e.g., pulmonary/renal tuberculosis) in a poor nutritional state that might affect the evaluation of clinical outcomes. The ethics review committee of the Shaanxi Provincial People's Hospital approved the study protocol (2012 - 0125). All patients provided written informed consent for the use and publication of data for research purposes.

Therapeutic Methods

Preoperative therapy

All patients diagnosed with thoracic spinal TB were treated with HREZ $(300 \mathrm{mg} / \mathrm{d}$ isoniazid, $450 \mathrm{mg} / \mathrm{d}$ rifampicin, $750 \mathrm{mg} / \mathrm{d}$ ethambutol and $1.5 \mathrm{~g} / \mathrm{d}$ pyrazinamide) for at least two to four weeks before the operations.

Operative technique

All patients were intubated under general anaesthesia. Patients were placed in a prone position, and a posteromedial incision was made to expose the vertebral plate, facet joints, and costovertebral joints. After the location of the lesion by C-arm fluoroscopy, pedicle screws were inserted in the normal vertebral body adjacent to the upper and lower affected vertebrae, followed by kyphosis correction. From the costovertebral joints or pedicles of the vertebral arch, the lesion in the anterior vertebral body was removed, as were the dead bone, necrotic intervertebral disc, and caseous necrotic tissue. Then, an autogenous iliac bone graft was embedded into the focal zone (Fig. 1). Following the operation, conventional bacterial cultivation and pathological diagnosis were performed.

Postoperative treatment

Prophylactic antibiotics were used for $72 \mathrm{~h}$, and oral administration of HREZ continued after the operation. Six months later, Pyrazinamide was withdrawn while isoniazid, rifampicin, and ethambutol (HRE) were administered daily for another 10 to 12 months. The drainage tube was removed when the 24$\mathrm{h}$ drainage volume was less than $50 \mathrm{ml}$. Following that, the patients were allowed to walk with the support of a mobility aid. At first, only non-weight-bearing activity was recommended. Normal weight- 
bearing activity was not allowed until the confirmation of intervertebral fusion with x-ray and CT examinations.

\section{Evaluation Standard}

Visual analogue scale (VAS) scores were used for pain assessment. The Frankel grading system was employed in the assessment of pre- and postoperative spinal cord injuries. ESR and CRP levels were assessed to monitor the disease activity. The pre- and postoperative kyphosis angles were measured using the method proposed by a previous study [12]. X-ray and CT examinations were conducted to assess bone graft fusion, loss of correction angle, and internal fixation failure [13].

Statistical analysis

The software SPSS 19.0 (SPSS, Inc., Chicago, IL, USA) was used for data analysis. Wilcoxon's signedrank test was used for assessing the differences between pre- and postoperative American Spinal Injury Association (ASIA) impairment scale scores. The paired t-test was performed to compare the pre- and postoperative degrees of kyphosis deformity and ESR and CRP levels. The Wilcoxon rank-sum test was employed in the analysis of discrepancy in normal distributions. P-value $<0.05$ was considered indicative of a significant difference.

\section{Results}

Definite diagnosis was established through bacterial cultivation and/or pathological diagnosis in all 88 cases, and mycobacterium tuberculosis was found in 66 patients. Out of the 88 patients, 50 were male and 38 were female; the mean age was $44.6 \pm 7.8$ years (range, $22-69$ ). The mean operation time was $165 \pm 38 \mathrm{~min}$. The mean intraoperative blood loss was $468 \pm 103 \mathrm{~mL}$. The mean length of hospital stay was $21 \pm 4 \mathrm{~d}$. The mean time of bone graft fusion was $6.6 \pm 0.3 \mathrm{~m}$. Varying degrees of paraspinal abscess around the muscle were observed in 14 patients. It took $7.2 \pm 0.6 \mathrm{~m}$ (range, 6-12 $\mathrm{m}$ ) for the abscess to disappear.

Follow-ups were performed in $100 \%$ of patients, with the mean duration of follow-up in the entire study population as $40.6 \pm 4.1 \mathrm{~m}$ (range, 36-48 m). Disruption of the wound occurred in one patient, and it healed $23 \mathrm{~d}$ after redressing and suture; there was a case of postoperative sinus track formation, and the sinus tract was treated properly and closed four weeks after the operation. A case of breakage of internal fixation was found during a follow-up eight months after the operation. During the follow-up period, no TB recurrence was reported.

Table 1 shows the pre- and postoperative kyphosis angles, angle correction, angle loss, and angle correction rates. The preoperative kyphosis angle was $26.5 \pm 4.5$. The postoperative and final-follow-up kyphosis angles were both significantly smaller than the pre-operative kyphosis angles $(P<0.05)$. The postoperative angle correction rate reached $81.5 \%$, and its postoperative angle loss was only $4.1 \%$. 


\begin{tabular}{|lllllll|}
\hline $\begin{array}{l}\text { Pre-operative } \\
\text { kyphosis } \\
\text { angle }\left({ }^{\circ}\right)^{*}\end{array}$ & $\begin{array}{l}\text { Post- } \\
\text { operation }\end{array}$ & & & $\begin{array}{l}\text { Final } \\
\text { follow-up }\end{array}$ & & \\
& Kyphosis & Angle & Correction & Kyphosis & Angle & Lost \\
& Angle $\left(^{\circ}\right)$ & Correction $\left({ }^{\circ}\right)$ & Rate $(\%)$ & Angle $\left(^{\circ}\right)$ & & rate $(\%)$ \\
& $5.6 \pm 1.9$ & $21.8 \pm 5.4$ & $\begin{array}{l}81.5 \pm \\
11.4\end{array}$ & $8.2 \pm 2.7$ & $1.1 \pm 1.0$ & $4.1 \pm 4.8$ \\
\hline $26.5 \pm 4.5$ & & & & & \\
\hline
\end{tabular}

Table 1. Kyphosis correction and kyphosis lost in single posterior approach

All patients had neurological impairment before operations (Frankel Grade C or D, Table 2). At the most recent follow-up, 82 patients had their neurofunctional parameters brought to within normal range. However, significant differences were found between the neurofunctional parameters before operations and at the final follow-up in each group $(P<0.05)$.

Table 2

Neurological recovery

according to Frankel grade

\begin{tabular}{|c|c|}
\hline Time point & $A B C D E$ \\
\hline Preoperative & 6424 \\
\hline Final follow-up* & \begin{tabular}{l|l|l} 
& 6 & 82 \\
\end{tabular} \\
\hline
\end{tabular}

Table 3 summarizes the changes in the VAS scores, ESR and CRP levels six weeks after operation and at the most recent follow-up. The VAS scores, ESR, and CRP levels six weeks after operations and at the final follow-up were significantly lower than the preoperative levels $(P<0.05)$. Beyond that, the VAS scores, ESR, and CRP levels were significantly lower than those recorded six weeks after operation $(\mathrm{P}<$ 0.05).

\section{Table 3}

Measures of surgical outcomes of the single posterior approach

\begin{tabular}{|l|l|l|l|l|l|l|}
\hline MeasureVAS & \multicolumn{1}{l|}{ CRP $(\mathrm{mg} / \mathrm{L})$} & \multicolumn{2}{|l|}{ ESR $(\mathrm{mm} / \mathrm{h})$} \\
\hline
\end{tabular}

\section{Discussion}

The existing treatment for spinal TB mainly includes anti-TB drugs and surgical intervention. Anti-TB drug therapy plays an essential role in treating spinal TB and provides a basis for surgical treatment. The goals of surgical treatment are to eradicate the lesion(s), relieve spinal cord compression, correct 
kyphosis deformity, and restabilize the spine [8]. Surgery alone, without regular anti-TB treatment, is extremely dangerous and ineffective. Effective surgical treatment is only possible in combination with effective anti-TB drug therapy. It is difficult to relieve spinal cord compression, improve nerve dysfunction and prevent progressive spinal deformity using conservative treatment alone. In contrast, surgery is an effective solution to this problem [14].

The choice of surgical approach to use when accessing the lesion sites remains a controversial issue. The single anterior approach has been widely accepted as the "gold standard" for spinal TB treatment [15] because it allows for thorough removal of lesions, decompression of the anterior structure of the spine, easy bone grafting, kyphosis correction, and reconstruction of spinal stability under direct vision, with the lesions being fully exposed [16]. However, it shows inadequate fixation rigidity and orthopaedic strength $[8,9]$ and imposes a high risk of vascular injury because of poor access to the lesion site [17]. Considering the high exposure, the large wound, and a great risk of hemopneumothorax, this method is not a good choice for patients with multisegmental thoracic spinal TB [18]. Other studies have found the anterior approach to be associated with postoperative kyphosis angle loss $[17,18]$.

As spinal TB surgical techniques develop, single posterior debridement, bone grafting, and instrumentation have achieved satisfactory outcomes of kyphosis correction and reconstruction of spinal stability, as well as long-segment fixation without creating a serious wound [10,11]. Zhang et al. [19] reported favourable clinical outcomes of thoracic spinal TB treatment using the single posterior approach. Liu et al. [20] performed posterior debridement, bone grafting, and instrumentation on patients with monosegmental thoracic spinal TB and achieved a satisfactory curative effect, with favourable bone graft fusion and significant improvement in the patients' Cobb angles. Hassan et al. [21] treated thoracic spinal TB patients with the anterior and posterior approaches and found that the posterior approach outperformed the anterior approach in mean operation time, intraoperative blood loss, and blood transfusion; in addition, the single posterior approach produced favourable surgical outcomes by creating a small wound to access the lesion site when treating thoracic spinal TB. In this study, the single posterior approach was performed on the 88 patients. Through analysis, these patients experienced significant improvement in their ASIA impairment scale scores and a sharp decrease in their Cobb angles and ESR levels after the operation. The pre- and postoperative parameters were significantly different $(P<0.05)$, indicating favourable surgical outcomes of the posterior approach. Furthermore, there was no significant difference between the two groups in the VAS scores and changes in ESR and ASIA impairment scale scores. However, the single posterior approach also has its own limitations. To be specific, it is difficult to remove TB lesions radically using the posterior approach; TB bacteria may invade the normal tissue in the posterior structure of the spine; the surgery may damage the normal posterior spine, compromise the spinal stability, interfere with the spinal cord and increase postoperative intraspinal scar adhesion.

Beyond that, this approach only gives limited exposure of the anterior structure of the spine, and thus it is not an option when there is a large paraspinal abscess. As stated in our previous study, the single posterior approach had a shorter operation time and a smaller volume of intraoperative blood loss compared with the anterior approach [8], and those findings were proven by this study. Through analysis, it is believed that these advantages of the posterior approach are associated with the development of the 
pedicle screw insertion technique and the posterior approach itself through day-to-day applications as well as the use of the approach in a large number of short-segment fixation (no more than three segments) cases. Additionally, the posterior approach had an angle loss rate smaller than that of the anterior approach in terms of kyphosis correction, which was reported in another relevant study [21]. The rationale for using the posterior approach lies in the removal of the lesion and the sclerotic bone surrounding the lesion to clear the path for anti-TB drugs. As to the residual TB-like lesion and purulent fluid, long-term, standard anti-TB chemotherapy is an effective postoperative solution. This study has some limitations, including the small sample size. A prospective multicentre randomized comparative study with a larger sample size is needed to investigate the long-term efficacy of the single posterior approach.

\section{Conclusions}

Single posterior debridement, bone grafting, and instrumentation combined with effective standard antiTB chemotherapy produce satisfactory clinical outcomes in treating thoracic spinal TB. In fact, the single posterior approach has many advantages, including a shorter operation time and a smaller volume of intraoperative blood loss than the single anterior approach; furthermore, it clearly outperforms the single anterior approach in kyphosis correction, angle correction rate, and angle loss. All bone grafts showed fusion. To date, the patients are in good condition according to the clinical and imaging results. Despite the preliminary results from the medium-term follow-up, it is necessary to perform a study based on longterm follow-up.

\section{Abbreviations}

Tuberculosis: TB; CT: Computed tomography; MRI: Magnetic resonance imaging; Pre-op: pre-operation; VAS: Visual analog scale; ESR: Erythrocyte sedimentation rate; CRP: C-reactive protein; Post-op: postoperation; ASIA: American Spinal Injury Association.

\section{Declarations}

Ethics approval and consent to participate

Ethical approval from the Ethics Committee of the Shaanxi Provincial People's Hospital was obtained for this study (No. 2012-0125). Each author certifies that all investigations were conducted in conformity with ethical principles. Written informed consent was obtained from all patients included in the study.

Consent for publish

All patients signed informed consent to publish their personal details in this article.

Availability of data and materials 
The datasets supporting the conclusions of this article are included within the article. The raw data can be requested from the corresponding author on reasonable request.

Competing interests

The authors declare that they have no competing interests.

Funding

Not applicable.

Authors' contributions

JZ, QL, QSand YZ participated in the recruitment, data collection and analysis. All authors contributed to the study design and drafting of the manuscript. All authors read and approved the final manuscript.

Acknowledgements

Not applicable.

\section{References}

1. Huang QS, Zheng $C, H u$ Y, Yin X, Xu H, Zhang G, Wang Q. One-stage surgical management for children with spinal tuberculosis by anterior decompression and posterior instrumentation. Int Orthop. 2009; 33( 5) : 1385-1390区

2. Garg RK, Somvanshi DS. Spinal TB: A review. J Spinal Cord Med 2011, 34: 440-454.

3. Dara M, Dadu A, Kremer K, et al. Epidemiology of TB in WHO European Region and public health response. Eur Spine J 2013, 22 Suppl 4:549-555.

4. Glaziou P, Floyd K, Raviglione MC. Global Epidemiology of Tuberculosis. SeminRespir Crit Care Med. 2018, 39(3):271-285.

5. Benli IT, Acarog lu E, Akalin S, Kis M, Duman E, Un A. Anterior radical debridement and anterior instrumentation in tuberculosis spondylitis. Eur Spine J. 2003; 12:224-234.

6. Jain AK, Aggarwal A, Dhammi IK, Aggarwal PK, Singh S. Extrapleural anterolateral decompression in tuberculosis of the dorsal spine. The Journal of bone and joint surgery British volume. 2004; 86 (7):1027-1031

7. Zhang P, Peng W, Wang X, Luo C, Xu Z, Zeng H, Liu Z. Minimum 5-year follow-up outcomes for singlestage transpedicular debridement, posterior instrumentation and fusion in the management of thoracic and thoracolumbar spinal tuberculosis in adults. Br J Neurosurg. 2016, 30(6):666-671.

8. Yongchun Zhou, Weiwei Li, Jun Liu, Liqun Gong, Jing Luo. Comparison of single posterior debridement, bone grafting and instrumentation with single-stage anterior debridement, bone grafting and posterior instrumentation in the treatment of thoracic and thoracolumbar spinal tuberculosis. BMC Surg. 2018, 3,18(1):71 
9. Yongchun Zhou, Zongrang Song, Jing Luo, Jijun Liu, Yunfei Huang, Yibin Meng, Wentao Wang, Dingjun Hao. The efficacy of local continuous chemotherapy and postural drainage in combination with one-stage posterior surgery for the treatment of lumbar spinal tuberculosis. BMC Musculoskelet Disorders, 2016,17(1):66

10. Sun L, Song Y, Liu L, Gong Q, Zhou C. One-stage posterior surgical treatment for lumbosacral tuberculosis with major vertebral body loss and kyphosis. Orthopedics. 2013, 36:e1082-90.

11. Li J, Li XL, Zhou XG, Zhou J, Dong J. Surgical treatment for spinal tuberculosis with bilateral paraspinal abscess or bilateral psoas abscess: one-stage surgery. .J Spinal Disord Tech. 2014, 27:E309-14.

12. Carman DL, Browne RH, Birch JG. Measurement of scoliosis and kyphosis radiographs. Intraobserver and interobser variation. The Journal of Bone Joint Surgery. 1990; 72(3):328-333.

13. Moon MS, Woo YK, Lee KS, Ha KY, Kim SS, Sun DH. Posterior instrumentation and anterior interbody fusion for tuberculosis kyphosis of dorsal and lumbar spines. Spine. 1995; 20(17):1910-1916.

14. Mak KC, Cheung KM. Surgical treatment of acute TB spondylitis: indications and outcomes. Eur Spine J.2013,22 (Suppl 4):603-611.

15. Benli IT, Kaya A, Acaroglu E. Anterior instrumentation in tuberculous spondylitis: is it effective and safe? Clinical orthopaedics and related research. 2007, 460:108-116.

16. Jin D, Qu D, Chen J, Zhang H. One-stage anterior interbody autografting and instrumentation in primary surgical management of thoracolumbar spinal tuberculosis. Eur Spine J. 2004, 13(2):114121.

17. Wang LJ, Zhang HQ, Tang MX, Gao QL, Zhou ZH, Yin XH. Comparison of Three Surgical Approaches for Thoracic Spinal tuberculosis in Adult: Minimum 5-Year Follow-Up. Spine (Phila Pa 1976). 2016; 42(11):808-817.

18. Assaghir YM, Refae HH, Alam-Eddin M. Anterior versus posterior debridement fusion for single-level dorsal tuberculosis: the role of graft-type and level of fixation on determining the outcome. Eur Spine J. 2016; 25(12):3884-3893.

19. Zhang HQ, Lin MZ, Shen KY, Ge L, Li JS, Tang MX, Wu JH, Liu JY. Surgical management for multilevel noncontiguous thoracic spinal tuberculosis by single-stage posterior transforaminal thoracic debridement, limited decompression, interbody fusion, and posterior instrumentation (modified TTIF). Arch Orthop Trauma Surg. 2012; 132:751-757.

20. Liu P, Sun M, Li S, Wang Z, Ding G. A retrospective controlled study of three different operative approaches for the treatment of thoracic and lumbar spinal tuberculosis: three years of follow-up $\mathbb{J} \mathbb{\text { V}}$. Clin Neurol Neurosurg $₫ 2015 \otimes 128 \llbracket 25-34$.

21. Hassan K®Elmorshidy E. Anterior versus posterior approach in surgical treatment of tuberculous

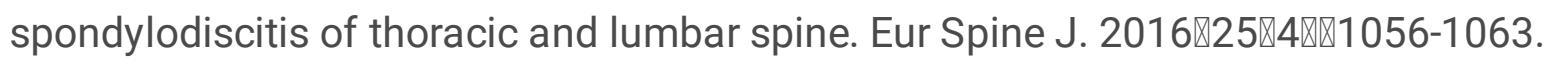

\section{Figures}




\section{Figure 1 is not available with this version of the manuscript}

\section{Figure 1}

The patient (female; 43 years old) presented with thoracic tuberculosis at T3-4 and underwent single posterior debridement, bone graft fusion, and instrumentation. $(a, b)$ : X-ray in the positive and lateral position before surgery. $(c, d)$ : preoperative $C T$ and MRI examination. $(e, f)$ : $X$-ray in the positive and lateral position after surgery. 